\title{
Automatic Quality and Moisture Evaluations Using Evolution Constructed Features
}

\author{
Meng Zhang ${ }^{1}$, Dah-Jye Lee ${ }^{1^{*}}$, Kirt Lillywhite $^{2}$, and Beau Tippetts ${ }^{2}$ \\ ${ }^{1}$ Dept. of Electrical and Computer Engineering, Brigham Young University \\ Provo, Utah 84602, USA \\ ${ }^{2}$ Smart Vision Works International, LLC., Orem, Utah 84058, USA \\ * Corresponding Author: Dah-Jye Lee
}

Email Address: djlee@ byu.edu, Telephone \#: +18014225923, Fax \#: 1+8014220201

Address: Brigham Young University, 459 CB, Provo, Utah 84602, USA

\section{Abstract}

Many quality evaluation tasks that are complicated and unique to specialty crops are often

carried out manually by human experts by visually inspecting product appearances. This labor-

intensive process usually depends greatly on experienced workers and lacks verification efficiency.

Automating these tasks not only reduces the processing time, improves the verification accuracy,

but also reduces the labor costs. This study is conducted to explore the feasibility of using our

recently developed Evolution-Constructed Feature (ECO-Feature) to automatically evaluate the quality of agricultural products. Three feature descriptors are added to the original ECO-Feature algorithm to reduce its dimensionality and improve training efficiency. This paper uses date fruit, cashews, pistachios, and almonds as examples to demonstrate the performance of the proposed algorithm for quality and moisture evaluations. The proposed method is proven accurate, effective and has been implemented and deployed for commercial production for the date 
industry in the United States.

\section{Keywords:}

Quality evaluation, Moisture evaluation, ECO-Feature, Object classification

\section{Introduction}

Quality evaluation of specialty crops is a challenging task. Quality is considered to be a combination of many different features. Some of these features are visual appearance (color, shape, size, defect), texture (crispness, firmness) and flavor (smell and taste) (Ignat et al., 2014). Evaluating the quality of specialty crops is important for producing high quality food products. Because of labor cost concerns, date fruit (especially Medjool) are harvested at varying levels of maturity in order to reduce the workload during the harvest season (Zhang et al., 2014a). Harvested dates must go through a post-harvest inspection, which classifies the dates according to their visual appearances. Ripe dates are sent for quality grading and packing. Less mature dates, on the other hand, have to go through a drying process which helps them ripen and prevents them from turning rotten or sour (Lee et al., 2008b). Date moisture must be evaluated accurately to determine the length of drying process because improper drying lowers the quality and increases food safety risks.

Almond, cashew and pistachio are three economically important nuts in the United States. They are all rich in nutrient and flavor with distinctive textures. These specialty crops are usually harvested at the mature stage and require proper drying and shell removal process. A major problem in the processing after harvesting is that nuts without shell are easily broken. Kernel completeness is one of the most important attributes consumers consider to determine product 
quality. Complete kernels have higher commercial values than broken ones. Evaluation of kernel completeness is necessary to satisfy consumer demands.

In general, product quality is evaluated by experienced workers, which is time-consuming and lacks efficiency (Clement et al., 2012). Automating complicated grading tasks will increase verification accuracy and help the producers of specialty crops to improve their competitiveness. To overcome these challenges, many visual appearance-based methods have been proposed to inspect fruits for tasks such as defect detection, quality grading, bruise damage measurement and variety classification (Brosnan et al., 2004; Nicolai et al., 2007; Opara et al., 2014). A hyperspectral imaging system was built to extract features for evaluating quality of apples (Xing et al., 2007). Reflective near-infrared imaging technique was used in the machine vision system to evaluate date fruit quality (Lee et al., 2008a). Among all kinds of visual features, color is an important quality attribute that dictates the quality and value of many fruit products (Kang et al., 2008). An image-dependent color quantization technique was designed specifically for real-time color evaluation in fruit maturity (Lee et al., 2008b). A novel color mapping concept for automated color grading was presented to specify and adjust settings for color preference (Lee et al., 2011). A multispectral vision system that is able to classify fresh-cut apple slices was developed to evaluate enzymatic browning (Lunadei et al., 2011). An efficient histogram analysis algorithm was proposed for real-time automated fruit surface quality evaluation (Zhang et al. 2014a and 2014b). Internal quality attributes of mango were accurately predicted by short-wave near-infrared spectroscopy and Fruit ripeness classification by NIRS calibrations showed accuracies between 59 and 88\% (Rungpichayapichet et al., 2016). An appropriately crafted mixture of fifteen different visual features was used in their computer vision based date 
classification system (Haidar et al., 2012).

Most of these existing visual appearance-based methods require a single characteristic such as color or a mixture of several hand-crafted features. These methods for quality evaluation usually depend on a human expert to determine or design the relevant features. Although these manually-selected features are able to describe the object of interest well and produce good accuracy for classification, specific features created by human experts that are good for one class of products often do not perform well for others. Features perform well on one product usually do not generalize well to other products. New features must be developed or grading parameters must be reset for different products or different varieties of the same product. This feature engineering process often involves a change of algorithm and software or fine-tuning of sorting parameters, which requires unique skills and extensive training.

We propose to use our recently developed Evolution Constructed Feature (ECO) algorithm (Lillywhite et al., 2012; Lillywhite et al., 2013) for quality grading. Unlike most visual inspection systems that use hand-crafted features, the ECO-Feature algorithm automatically discovers salient features from a training dataset without the involvement of a human expert. It is a fully automated feature construction method. Rather than relies on a human expert to design or construct features, the proposed method uses standard genetic algorithm (Mitchell, 1998) to get high quality features from the input images. The proposed method is capable of constructing non-intuitive features that are often overlooked by the human experts. Its unique capability allows easy adaption of this technology for various specialty crops that require accurate classification of products when the differentiation between them is not defined or cannot be well described. 
We further improve the effectiveness of ECO-Feature by using histogram-based feature descriptors in this work. It is known that the original ECO feature is sensitive to even a small shift or rotation of the object in the image and the feature vector has high dimensionality. The high dimensionality leads to high computational complexity for the classifiers. A better approach of calculating the feature vector is needed to overcome these limitations of the original ECO-Feature. In this study, we propose to represent ECO features with descriptors. Using descriptors not only captures the essential properties of ECO-Feature but also provides property of being invariant to image deformations. They are able to extract useful information that is robust to transforms, rotation or lighting change of objects (Mikolajczyk and Schmid, 2005). Additionally, using descriptors allows us to improve ECO-Feature training speed as feature dimensionality on average is reduced (Zhang \& Lee, 2015). In this improved ECO-Feature algorithm, we include three histogram-based feature descriptors including Histograms of Oriented Gradients (HOG) (Dalal et al., 2005), Local Binary Patterns Histograms (LBPH) (Ahonen et al., 2004) and a histogram of the Hu set $(\mathrm{Hu}, 1962)$ values of images.

\section{Materials and methods}

\subsection{Sample collection}

We collected samples of dates, cashews, pistachios, and almonds to generate a dataset for our experiments in order to prove the improved ECO-Feature works well for quality evaluation and the use of feature descriptors helps ECO-Feature improve its classification efficiency.

Dates used in this study were harvested Medjool dates which are usually sorted into four classes of maturity according to their color (Zhang et al., 2014b). Figure 1 shows samples of 
Medjool dates taken from these four classes. Dates in light red and orange (the middle two samples in Figure 1) are usually considered to have the same maturity level but with different moisture content. They have very similar colors. Color grading is often not accurate on these two types of dates. In this paper, we focus our work on classifying these two types of dates which have the same maturity level but different moisture content.
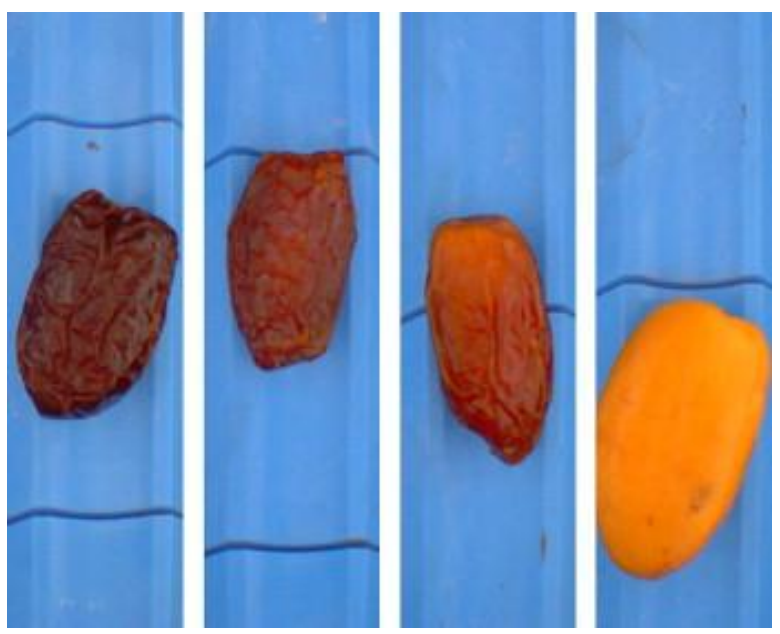

Figure 1. Samples of Medjool dates. From left to right, it shows dates of dark red (mature), light red (slightly moist), orange (wet), and yellow (not ripe).

Our date dataset is with two moisture levels, dry and wet. Each moisture level contains 400 dates which were selected by human experts for our experiments. This human grading result was considered to be the ground truth in order to evaluate the performance of automatic grading based on our ECO-Feature method. Eight sample images are shown in Figure 2.
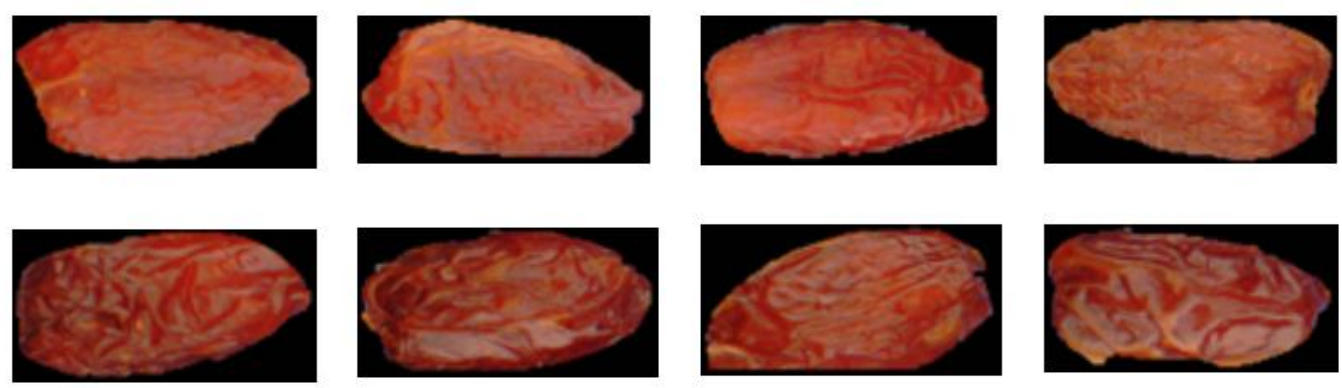

Figure 2. Samples from the date dataset. The top row shows the dry date samples. The bottom row shows the wet date samples. 
experiments for quality evaluation. For each dataset, we separated complete nuts from broken are shown in Figure 3.
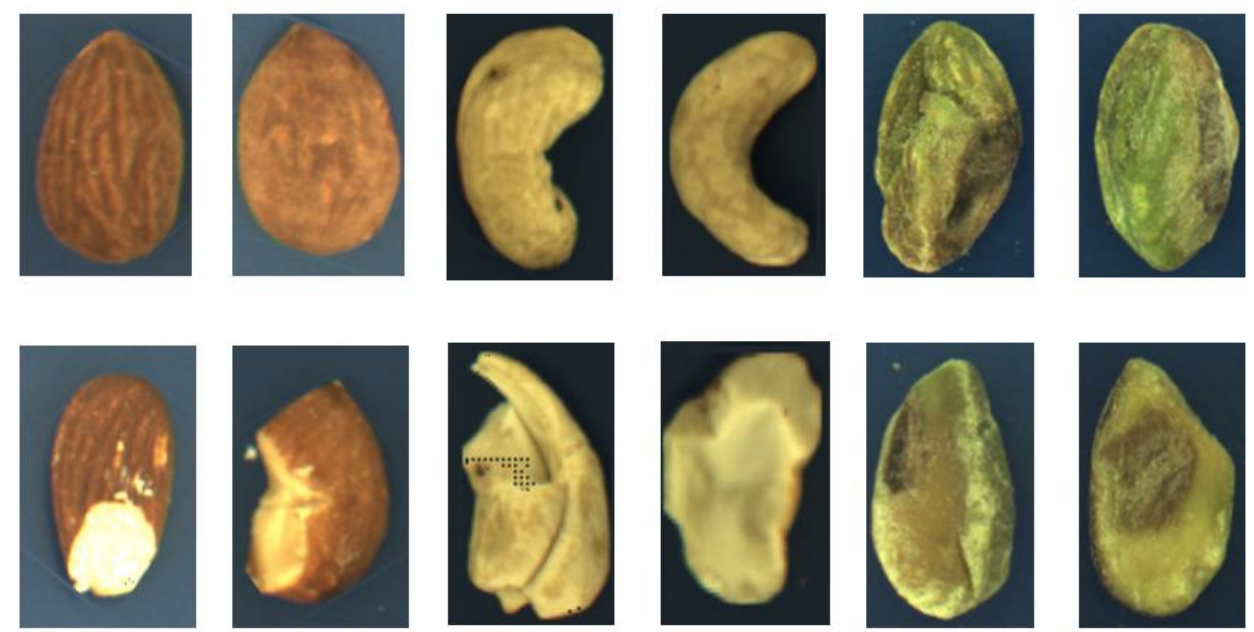

136

137

138

139

Figure 3. Samples of complete nuts (almond, cashew, and pistachio) are shown in the top row. Samples of broken nuts are shown in the bottom row.

\subsection{System Design}

The proposed method has been implemented in a commercial inspection system to classify harvested dates according to their color. Figure 4 (a) shows the overall view of the inspection system. The harvested dates are first arranged into single file by a singulator and delivered onto a U-shaped plastic carrier (See Figure 4 (b)). The carrier transfers the singlulated dates into a light chamber for imaging. Six Firewire color cameras (Point Grey Flea-2) with 640×480-pixel resolution are housed in the light chamber which provides controlled and consistent illumination. Twelve image 

system

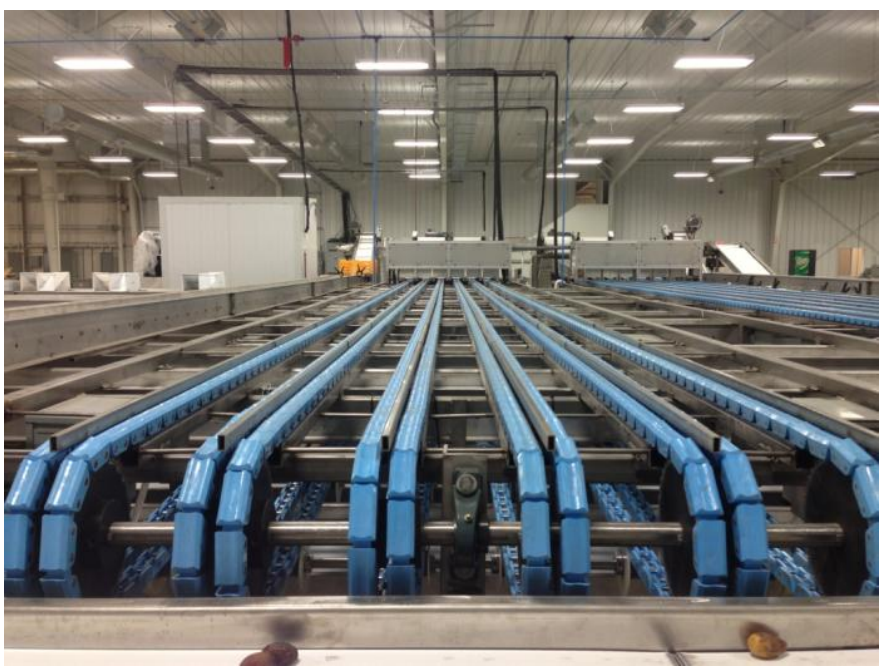

(a)

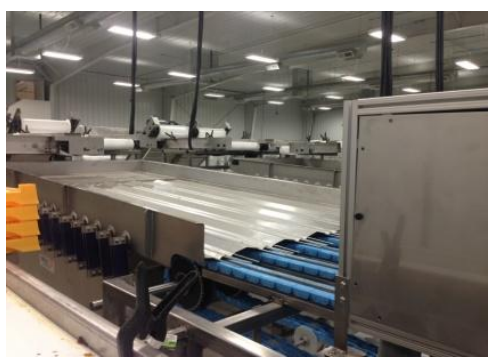

(b)

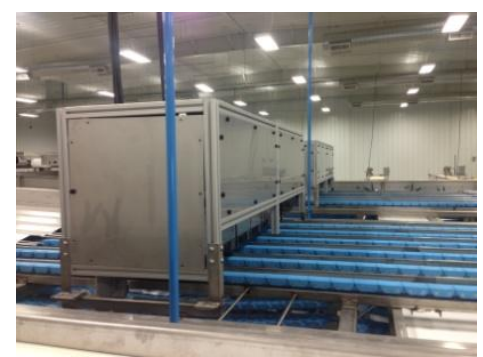

(c)

Figure 4. Photographs of a 12-lane system. (a) the overall view of the system; (b) singulation section that singulates fruit into single files; (c) light chamber that houses the camera and 


\subsection{Automatic feature construction}

\subsubsection{ECO-Feature Construction}

ECO-Feature (Lillywhite et al., 2012; Lillywhite et al., 2013) is an effective method for automatic feature construction. Most hand-crafted feature algorithms make assumptions about where the good features can be found. Unlike those manually designed features, ECO features are automatically constructed using a standard genetic algorithm (GA). A GA is a search algorithm based on the idea of natural selection process. GAs simulate the survival of the fittest among individuals over generations and are generally used to solve optimization problems. Each generation in GAs consists of a population of individuals, which represent possible solutions to a specific problem. Over generations of crossover (recombining between individuals) and mutation (getting modified inside each individual) within simulated evolution, the individuals in the population evolve to be fitter than the initial population.

In our work, each individual in GA represents an ECO-Feature that can be used for our object classification tasks. The parameters of an ECO-Feature are the location of this feature, the transforms that it consists of, and the parameters for each corresponding transform. GA helps search for the parameters with which these features will have the best classification performance. In this way, how good an ECO feature could be on the selected sub-region is determined by its own ability to help classify objects rather than a human expert's assumption. Both global and local information of objects can be captured in each ECO feature. Each ECO feature specializes in representing different aspects of the object.

The GAs start with a population of ECO features with their initial parameter settings. During the construction of ECO features, a fitness score is computed for each ECO feature in the 


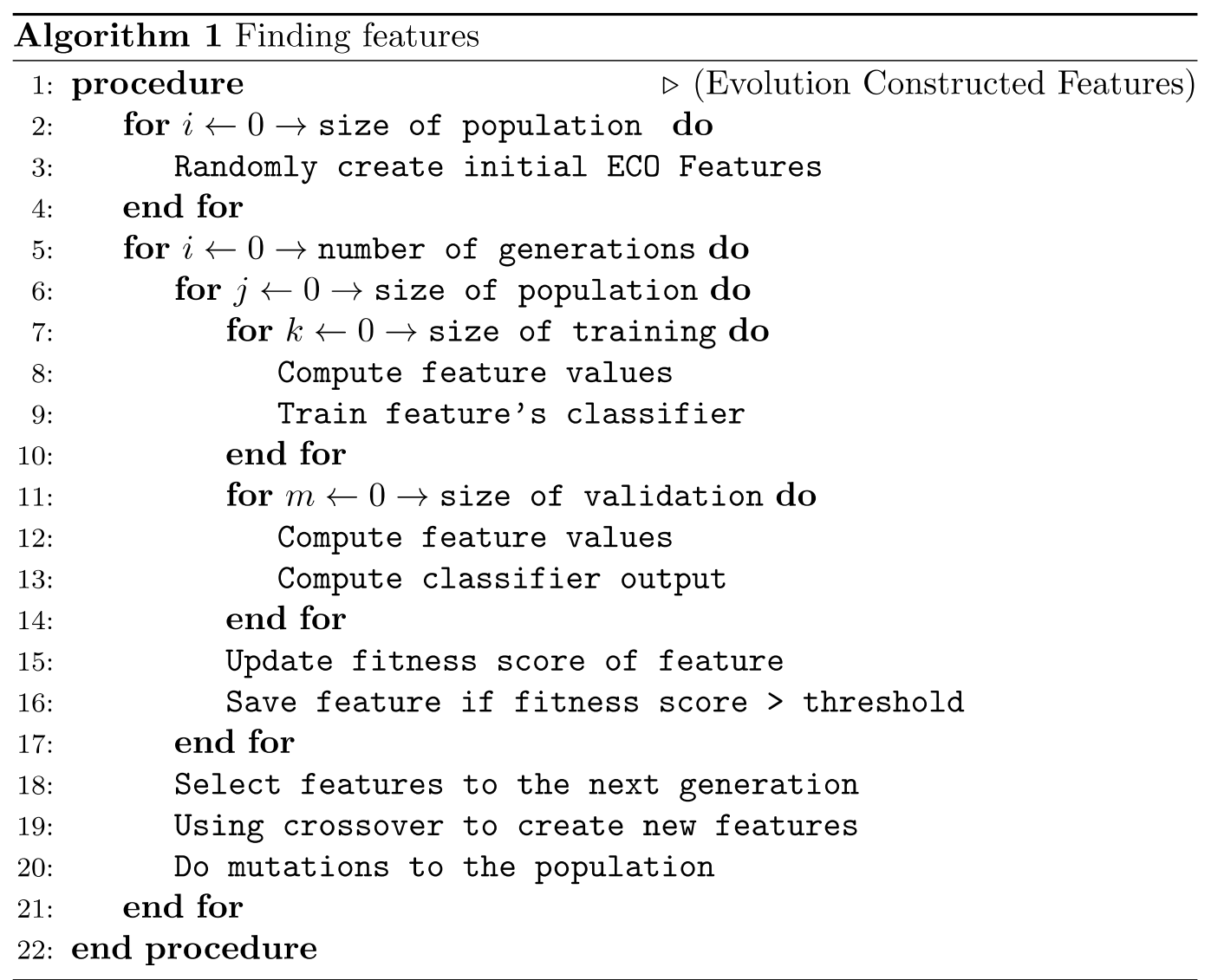

Algorithm 1. Finding ECO-Feature. 


$$
\delta_{M}[m]=\frac{\delta_{\mathrm{M}}[\mathrm{m}] * \mathrm{e}^{-\rho \cdot \mathrm{c}}}{\text { Normalization Factor }}
$$

In Equation $2, \delta_{M}[\mathrm{~m}]$ is the weight for each image, $\rho$ is calculated in Equation 1 and $\mathrm{c}$ is the output of each weak classifier defined in Equation 3.

$$
\mathrm{C}=\left\{\begin{aligned}
1, & \text { if classified corectly } \\
-1, & \text { else }
\end{aligned}\right.
$$




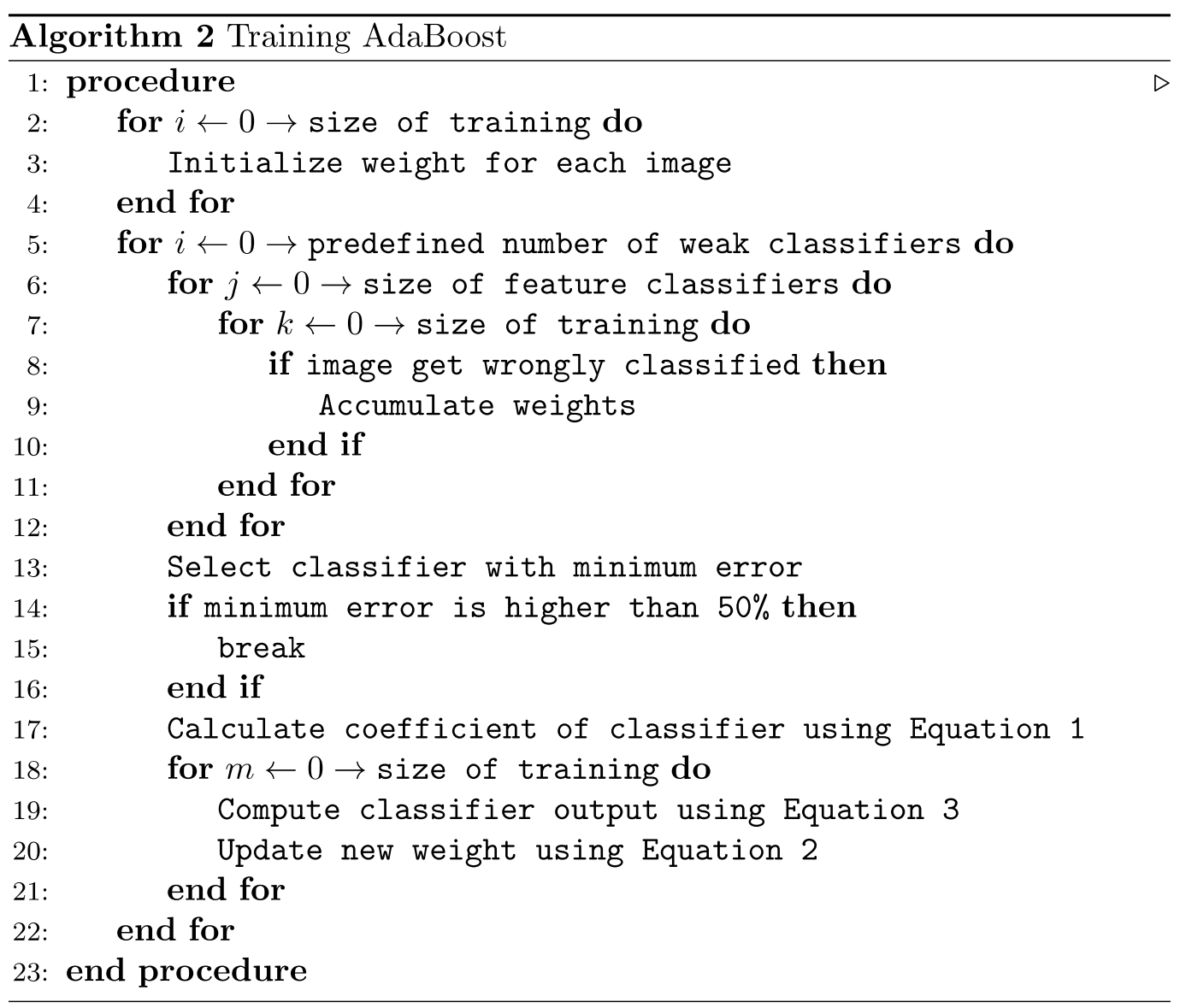

Algorithm 2. Training AdaBoost.

\subsection{Moisture and quality evaluation}

Figure 5 shows an example of classifying an image with an AdaBoost model containing two

ECO features. Each ECO feature operates on its own sub-region of the image. As the ECO feature

222 one transform to the next, just as the two ECO features shown in Figure 5. The number of the 
Image Image Transforms

Descriptor

AdaBoost
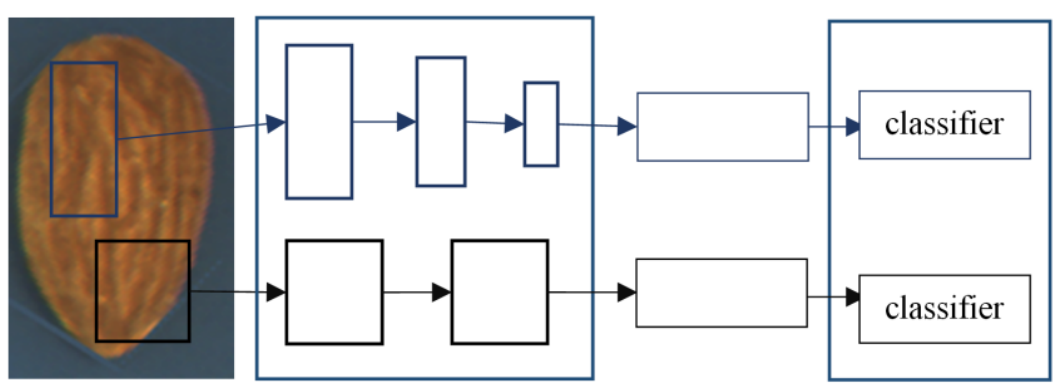

Figure 5. ECO-Features and their corresponding classifiers are combined using AdaBoost to classify an image.

The output of each weak classifier is combined according to Equation 4 where $X$ is the number of weak classifiers in the AdaBoost model, $\rho_{\mathrm{x}}$ is the coefficient for weak classifier $\mathrm{x}$ (see Equation 1), $\alpha_{\mathrm{x}}$ is the output of the weak classifier, $\tau$ is a threshold value, and $\mathrm{c}$ is the final classification given by the AdaBoost model. The threshold $\tau$ can be selected to adjust the tradeoff between false positives and false negatives. Different thresholds can be selected to adjust the classification performance for specific applications. Accepting more true positives will inevitably increase false positives (or fewer true negatives) in the classification result.

$$
c=\left\{\begin{array}{l}
1 \text { if } \sum_{x=1}^{x} \rho_{x} \cdot \alpha_{x}>\tau \\
0 \quad \text { else }
\end{array}\right.
$$

\subsection{Evaluation metrics}

We construct ECO features on the training set and then evaluate them using the testing set. The classification accuracy, precision and recall are computed according to the following equations (Equation 5 and 6):

$$
\text { precision }=\frac{\text { true positive }}{\text { true positive }+ \text { false positive }}
$$




$$
\text { recall }=\frac{\text { true positive }}{\text { true positive }+ \text { false negative }}
$$

\section{Results and Discussion}

\subsection{Training and testing}

We performed experiments on our date dataset and nut datasets using both the original

ECO-Feature and the improved ECO-Feature. For each dataset, we randomly chose $75 \%$ of the images as the training set and $25 \%$ of the images as the testing set. During the construction of ECO features, a population size of 50 was used in the genetic algorithm to search for the best feature that can be used for classification. We believe using this population size allows the genetic algorithm to explore more possible combinations of image transforms and thus achieve diversity in the constructed features. And the training ends either when a satisfied fitness score is obtained or the best fitness score for each training iteration remains the same for several consecutive iterations. From our experiments, we determined that using these criteria to terminate the training process allows more efficient construction of ECO features and is better than using a predefined number of generations. classification model. Setting the threshold allows us to determine a desired tradeoff between false positive and false negative. In our experiments on the datasets, we chose the thresholds which gave the highest classification accuracy to report our results.

\subsection{Performance Evaluation}


objectives in this work. All three feature descriptors selected for our ECO-Feature were able to significantly reduce the dimensionality of an ECO feature. In our experiments, we divided the input image into $2 \times 2$ blocks and computed a histogram from these blocks. When using HOG as the descriptor, we computed a gradient direction histogram over eight directions and the histograms were concatenated to form a feature vector. For LBPH feature descriptor, we first calculated a local binary pattern image from the input image to have each pixel value set between 0 and 8 . And then we extracted a histogram from each block of the image. The use of feature descriptors greatly reduced the computational cost for training classifiers. For a $50 \times 50$ image region, the dimensionality of the original ECO feature could be as large as 2500 while the dimensionality of ECO-Feature when including HOG as its descriptor was reduced to 32 and for the other two feature descriptors the feature dimensionality was reduced to 36 . By using feature descriptors in ECO-Feature, the feature dimensionality could be greatly reduced and thus the classifiers associated with each feature can be trained efficiently.

\subsubsection{Quality evaluation}

The performances on the pistachio, almond and cashew datasets are shown in Tables 1-6.

Our experimental results show that ECO-Feature obtained a classification accuracy of higher than $95 \%$ on the three datasets of nuts which demonstrated that our ECO-Feature is a good approach for quality evaluation of nuts. The highest classification accuracy of $100 \%$ was achieved on the almond dataset. The classification accuracy we obtained for pistachios was $98.0 \%$. The classification accuracy for the cashew dataset was lower than the other two datasets. After analyzing those misclassified images, we attributed this low accuracy to the similarity between the whole and broken cashews. Some samples were slightly broken and were hard to identify 
even by humans. Additionally, the shape of cashew varies in the dataset. Some sample images were from the front view and some were from the side view. This dataset is a challenge to our ECO-Feature considering that ECO-Feature did not perform well because of the variation caused by viewpoint change.

The performance on the pistachio dataset is shown in Tables 1-2. Table 1 shows the overall classification performance on the pistachio dataset. For pistachios, the original feature could achieve the classification accuracy of $98.0 \%$. When using LBPH in the ECO-Feature, the classification accuracy remains as good as the original feature. The accuracy was not improved but was not affected either after the feature dimensionality was reduced significantly to a fixed length (Zhang \& Lee, 2015). But when using HOG and statistic values in our ECO features, the classification accuracy was a little bit lower than the original ECO-Feature algorithm. The classification accuracy was lowered from $98.0 \%$ to $96.1 \%$, however, the dimensionality of features was reduced greatly to a fixed length. It can be considered as an acceptable sacrifice considering the feature dimensionality reduction.

Table 1. Experimental results and comparison to the original method on the pistachio dataset.

\begin{tabular}{|l|c|c|c|}
\hline & Classification rate & Precision & Recall \\
\hline Original feature & $98.0 \%$ & $97.6 \%$ & $100.0 \%$ \\
\hline With HOG & $96.1 \%$ & $95.3 \%$ & $100.0 \%$ \\
\hline With Statistics & $96.1 \%$ & $95.3 \%$ & $100.0 \%$ \\
\hline With LBPH & $98.0 \%$ & $97.6 \%$ & $100.0 \%$ \\
\hline
\end{tabular}


Table 2. Confusion matrix on pistachio dataset ( $F P=F a l s e$ positive, $F N=F a l s e$ negative, $T P=T r u e$ positive, $\mathrm{TN}=$ True negative).

\begin{tabular}{|l|c|c|c|c|}
\hline & FP & FN & TP (whole) & TN (broken) \\
\hline Original feature & 1 & 0 & 41 & 9 \\
\hline With HOG & 2 & 0 & 41 & 8 \\
\hline With Statistics & 2 & 0 & 41 & 8 \\
\hline With LBPH & 1 & 0 & 41 & 9 \\
\hline
\end{tabular}

The performance on almond dataset is shown in Tables 3-4. Table 3 shows the overall

classification performance on the almond dataset and the comparison with the original method.

Fifty-six samples ( $25 \%$ of 226 samples) were used for testing ( 29 whole and 27 broken). For

almonds, we obtained $100 \%$ classification accuracy on original feature as well as on the three

this dataset. The confusion matrix of the testing dataset is shown in Table 4.

Table 3. Experimental results and comparison to the original method on the almond dataset.

\begin{tabular}{|l|c|c|c|}
\hline & Classification rate & Precision & Recall \\
\hline Original feature & $100 \%$ & $100 \%$ & $100 \%$ \\
\hline With HOG & $100 \%$ & $100 \%$ & $100 \%$ \\
\hline With Statistics & $100 \%$ & $100 \%$ & $100 \%$ \\
\hline With LBPH & $100 \%$ & $100 \%$ & $100 \%$ \\
\hline
\end{tabular}

Table 4. Confusion matrix on the almond dataset (FP=False positive, $F N=$ False negative, $T P=$ True positive, $\mathrm{TN}=$ True negative).

\begin{tabular}{|l|c|c|c|c|}
\hline & FP & FN & TP (whole) & TN (broken) \\
\hline Original feature & 0 & 0 & 29 & 27 \\
\hline With HOG & 0 & 0 & 29 & 27 \\
\hline With Statistics & 0 & 0 & 29 & 27 \\
\hline With LBPH & 0 & 0 & 29 & 27 \\
\hline
\end{tabular}


Table 5. Experimental results and comparison to the original method on the cashew dataset.

\begin{tabular}{|c|c|c|c|}
\hline & Classification rate & Precision & Recall \\
\hline Original feature & $96.2 \%$ & $96.4 \%$ & $96.4 \%$ \\
\hline With HOG & $92.5 \%$ & $92.9 \%$ & $92.9 \%$ \\
\hline With Statistics & $96.2 \%$ & $96.4 \%$ & $96.4 \%$ \\
\hline With LBPH & $92.5 \%$ & $96.2 \%$ & $89.3 \%$ \\
\hline
\end{tabular}

three samples ( $25 \%$ of 214 samples) were used for testing ( 28 whole and 25 broken). The confusion matrix on this dataset is shown in Table 6. For cashews, when statistic values were used in the ECO features, the classification accuracy was the same as the original ECO-Feature algorithm. When having HOG and LBPH included in the features, the classification accuracies were slightly lower than the original ECO-Feature algorithm, reduced from $96.2 \%$ to $92.5 \%$. It can be considered as an acceptable decrease in performance when considering the great feature dimensionality reduction.

Table 6. Confusion matrix on the cashew dataset ( $F P=F a l s e$ positive, $F N=F a l s e$ negative, $T P=$ True positive, $\mathrm{TN}=$ True negative).

\begin{tabular}{|l|c|c|c|c|}
\hline & FP & FN & TP (whole) & TN (broken) \\
\hline Original feature & 1 & 1 & 27 & 24 \\
\hline With HOG & 2 & 2 & 26 & 23 \\
\hline With Statistics & 1 & 1 & 27 & 24 \\
\hline With LBPH & 1 & 3 & 25 & 24 \\
\hline
\end{tabular}

\subsubsection{Moisture evaluation}

As mentioned in the previous section, we aimed to focus on our work on classifying the two types of dates that have the same maturity level but with different moisture. We used 100 pieces of dates with more moisture and 100 pieces of dates with less moisture for our experiments. Other types of maturity levels can be easily evaluated by using color information. In the date experiments, we evaluated the moisture of two types of dates in our dataset. When using LBPH 
in the ECO features, the classification accuracy was exactly the same as the original ECO-Feature, being $98.0 \%$. When using HOG and statistic values in the features, the classification accuracy was reduced from $98.0 \%$ to $96.5 \%$. The classification accuracy for the date dataset and the comparison to the original method is given in Table 7. More detailed experimental results are shown in Table 8.

Table 7. Comparison to the original method on date dataset.

\begin{tabular}{|l|c|c|c|}
\hline & Classification rate & Precision & Recall \\
\hline Original feature & 98.0 & 96.2 & 100 \\
\hline With HOG & 96.5 & 96.0 & 97.0 \\
\hline With Statistics & 96.5 & 96.0 & 97.0 \\
\hline With LBPH & 98.0 & 97.1 & 99.0 \\
\hline
\end{tabular}

Table 8. Confusion matrix on the date dataset (FP=False positive, $F N=$ False negative, $T P=T r u e$ positive, $\mathrm{TN}=$ True negative).

\begin{tabular}{|l|c|c|c|c|}
\hline & FP & FN & TP (moist) & TN (not moist) \\
\hline Original feature & 4 & 0 & 100 & 96 \\
\hline With HOG & 4 & 3 & 97 & 96 \\
\hline With Statistics & 4 & 3 & 97 & 96 \\
\hline With LBPH & 3 & 1 & 99 & 97 \\
\hline
\end{tabular}

\section{Conclusion}

In this paper, we have explored using our ECO-Feature algorithm to automatically evaluate quality of specialty crops including pistachios, almonds, cashews, and dates. The proposed ECOFeature algorithm presents an opportunity to automate quality evaluation tasks. It is designed to perform object classification without requiring human experts to define what are the good features and what parameters the features perform best with. Quality evaluation of specialty crops is time-consuming and inefficient and requires experienced workers. ECO-Feature has been demonstrated to be an ideal approach to overcome these technical challenges for quality 
verification applications. Our experimental results show that ECO-Feature performs very well for moisture evaluation and quality verification. It can be easily adapted to various product grading applications including but not limited to specialty crops such as plums, grapes, apples, pears, peaches, and peanuts.

In this work, we further improve the effectiveness of ECO-Feature by using feature descriptors. We propose to apply a small descriptor that captures essential properties of our ECO-Feature. Three feature descriptors are tested. The dimensionality of original ECO-Feature is greatly reduced. The classification performance of our original ECO-Feature is improved through using feature descriptors. From the pixel intensity representation in the original ECO-Feature to the feature descriptor representation, images are represented more compactly, which makes construction of ECO features more efficient. Since our original ECO-Feature is sensitive to small variations of object location and orientation, using feature descriptors in ECO-Feature not only allows us to capture the robust information of images but also makes ECO-Feature training faster. Feature descriptor representation is shown to provide good performance improvement over the original ECO-Feature algorithm. The proposed method is proven accurate and has been implemented and deployed for commercial production for the date industry in the United States

\section{Acknowledgment}

The project was supported by the Small Business Innovation Research program of the U.S. Department of Agriculture, grant number \#2015-33610-23786.

\section{References}

Ahonen, T., Hadid, A., \& Pietikäinen, M. (2004). Face recognition with local binary patterns. 
In Computer vision-eccv 2004 (pp. 469-481). Springer Berlin Heidelberg.

Brosnan, T., \& Sun, D. W. (2004). Improving quality inspection of food products by computer vision-a review. Journal of Food Engineering, 61(1), 3-16.

Clement, J., Novas, N., Gázquez, J. A., \& Manzano-Agugliaro, F. (2012). High speed intelligent classifier of tomatoes by colour, size and weight.Spanish Journal of Agricultural Research, 10(2), 314-325.

Dalal, N., \& Triggs, B. (2005, June). Histograms of oriented gradients for human detection. In Computer Vision and Pattern Recognition, 2005. CVPR 2005. IEEE Computer Society Conference on (Vol. 1, pp. 886-893). IEEE.

Haidar, A., Dong, H.W., Mavridis, N., (2012) “Image-based date fruit classification,” Proc. of IEEE Int.I Congress on Ultra Modern Telecommunications and Control Systems (ICUMT 2012), 369-375.

Hu, M. K. (1962). Visual pattern recognition by moment invariants. Information Theory, IRE Transactions on, 8(2), 179-187.

Ignat, T., Alchanatis, V., \& Schmilovitch, Z. E. (2014). Maturity prediction of intact bell peppers by sensor fusion. Computers and Electronics in Agriculture, 104, 9-17. 
401

402

403

404

405

406

407

408

409

410

411

412

413

414

415

416

417

418

419

420

421

422

Kang, S. P., East, A. R., \& Trujillo, F. J. (2008). Colour vision system evaluation of bicolour fruit: A case study with 'B74’mango. Postharvest Biology and Technology, 49(1), 77-85.

Lee, D. J., Schoenberger, R., Archibald, J., \& McCollum, S. (2008a). Development of a machine vision system for automatic date grading using digital reflective near-infrared imaging. Journal of Food Engineering, 86(3), 388-398.

Lee, D. J., Y. C. Chang, J.K. Archibald, and C.R. Greco, (2008b) “Color Quantization and Image Analysis for Automated Fruit Quality Evaluation," IEEE Conf. on Automation Science and Engineering (CASE), 194-199, Washington DC, USA.

Lee, D.J., J.K. Archibald, and G.M. Xiong, (2011) “Rapid Color Grading for Fruit Quality Evaluation Using Direct Color Mapping," IEEE Trans. on Automation Sci. and Eng., vol. 8(2), 292-302.

Lillywhite, K., Tippetts, B., \& Lee, D. J. (2012). Self-tuned Evolution-COnstructed features for general object recognition. Pattern Recognition, 45(1), 241-251.

Lillywhite, K., Lee, D. J., Tippetts, B., \& Archibald, J. (2013). A feature construction method for general object recognition. Pattern Recognition, 46(12), 3300-3314.

Lunadei, L., Galleguillos, P., Diezma, B., Lleó, L., \& Ruiz-Garcia, L. (2011). A multispectral vision system to evaluate enzymatic browning in fresh-cut apple slices. Postharvest Biology and 
Mitchell, M. (1998). An introduction to genetic algorithms. MIT press. review," Postharvest Biology and Technology, 46 (2) 99-118. mango. Postharvest Biology and Technology,111, 31-40. Donter Society Conference on (Vol.1, pp. I-511). IEEE.

Mikolajczyk, K., \& Schmid, C. (2005). A performance evaluation of local descriptors. Pattern Analysis and Machine Intelligence, IEEE Transactions on, 27(10), 1615-1630.

Nicolai, B. M., Beullens, K., Bobelyn, E., Peirs, A., Saeys, W., Theron, K. I., and Lammertyn, J. (2007) "Nondestructive measurement of fruit and vegetable quality by means of NIR spectroscopy: A

Opara, U. L., \& Pathare, P. B. (2014). Bruise damage measurement and analysis of fresh horticultural produce-A review. Postharvest Biology and Technology, 91, 9-24.

Rungpichayapichet, P., Mahayothee, B., Nagle, M., Khuwijitjaru, P., \& Müller, J. (2016). Robust NIRS models for non-destructive prediction of postharvest fruit ripeness and quality in

Viola, P., \& Jones, M. (2001). Rapid object detection using a boosted cascade of simple features. In Computer Vision and Pattern Recognition, 2001. CVPR 2001. Proceedings of the 2001 IEEE 
445

446

447

448

449

450

451

452

453

454

455

456

Xing, J., Saeys, W., and Baerdemaeker, J. D. (2007) “Combination of chemometric tools and image processing for bruise detection on apples," Computers and Electronics in Agriculture, 56(1), 1-13.

Zhang, D., D.J. Lee, K.D. Lillywhite, and B.J. Tippetts, (2014a) "Date Quality Evaluation Using Short-wave Infrared Imaging," J. of Food Eng., vol. 141, p. 74-84.

Zhang, D., D.J. Lee, K.D. Lillywhite, and B.J. Tippetts, (2014b) “Date Maturity and Quality

Evaluation Using Color Distribution Analysis and Back Projection," J. of Food Eng., vol. 131, p. 161169.

Zhang, M., \& Lee, D. J. (2015). Efficient Training of Evolution-Constructed Features.

In International Symposium on Visual Computing (pp. 646-654). Springer International Publishing. 